\title{
Pontas bifaciais no Brasil Meridional: caracterização estatística das formas e suas implicações culturais
}

\author{
Mercedes Okumura* \\ Astolfo Araujo*
}

\begin{abstract}
OKUMURA, M.; ARAUJO, A. Pontas bifaciais no Brasil Meridional: Caracterização estatística das formas e suas implicações culturais. R. Museu Arq. Etn., São Paulo, n. 23, p. 111-127, 2013.
\end{abstract}

Resumo: A Tradição Umbu, distribuída na porção sul e sudeste do Brasil, caracteriza-se pela presença de pontas bifaciais e apresenta datas desde 12.700 anos AP até o período histórico. Essa tradição é problemática por conta de sua ampla distribuição geográfica e cronológica. Este trabalho visa contribuir para a caracterização da variabilidade morfológica das pontas através de análises quantitativas. Os resultados mostram que os grupos de São Paulo manufaturavam pontas distintas daquelas dos grupos mais meridionais (Paraná, Santa Catarina e Rio Grande do Sul). Haveria, portanto, uma identidade regional compartilhada exclusivamente por alguns grupos paulistas (ao menos no que diz respeito às pontas). É possível que a Tradição Umbu seja mais restrita, tanto em termos cronológicos quanto em termos regionais, do que se propõe atualmente.

Palavras-chaves: Líticos, Morfometria geométrica, Tradição Umbu

\section{Introdução}

rtefatos líticos lascados em formato de A ponta são bastante comuns nas Américas, sendo denominados genericamente "pontas de flecha" ou "pontas de projétil". Embora sua função perfurante seja óbvia, podem ser vários os meios pelos quais a perfuração é produzida: pressão induzida pela força muscular de quem manuseia o artefato, transmitida diretamente

(*) Museu de Arqueologia e Etnologia, Universidade de São Paulo, Brasil <okumuram@usp.br><astwolfo@usp.br> por meio de uma haste (lança); pressão induzida pelo choque entre o artefato e o alvo, sendo a energia cinética produzida por meio de algum dispositivo (arco ou propulsor de dardos). Por fim, algumas pontas podem ter sido utilizadas como furadores, cuja ação se dá por meio de movimentos circulares acompanhados de pressão. Em suma, os termos "ponta de flecha" ou "ponta de projétil" são inadequados, uma vez que pressupõem um determinado meio de transmissão de energia em detrimento de outros. Sendo assim, designaremos tais artefatos como "pontas bifaciais".

Uma característica importante das pontas bifaciais reside no fato de as mesmas se 
constituírem em artefatos formais, ou seja, instrumentos cuja manufatura visa à obtenção de uma forma específica. No Brasil, um dos melhores exemplos de produção de pontas bifaciais é a denominada Tradição Umbu. Essa tradição, distribuída na porção sul e sudeste do país, foi caracterizada justamente pela presença de pontas bifaciais, apresentando idades desde 12.700 AP até o período histórico (Schmitz et al. 1980; Schmitz 1999; Noelli, 1999-2000).

São duas as hipóteses a respeito da origem da Tradição Umbu; uma delas supõe que a mesma teria se originado a partir da Fase Uruguai, já presente no $11^{\circ}$. milênio AP no sudoeste do Rio Grande do Sul (Kern 1981:232-8; Schmitz 1984:8-12; 1987). Outra hipótese seria a origem dessa Tradição a partir da Fase Vinitu encontrada no sudoeste do Paraná (Kern 1981: 215-220; Schmitz 1984:12-14). Schmitz (1987) chama a atenção para o fato de que, apesar do grande número de sítios identificados como representantes da tradição Umbu, pouquíssimos indicadores cronológicos têm sido identificados. Isso acabou por gerar controvérsias a respeito de sua própria existência, uma vez que tal "tradição" abarcaria uma faixa cronológica demasiadamente extensa, de 11.000 a 500 anos AP. Em áreas onde os sítios associados à Tradição Umbu são abundantes, como o sudeste do Paraná e o nordeste (e possivelmente o sudoeste) do Rio Grande do Sul, a cronologia parece ser bastante recuada. Já no planalto do Rio Grande do Sul, em Santa Catarina e no sul de São Paulo, tal tradição parece ser mais recente.

A Tradição Umbu coloca um problema especial aos arqueólogos por conta de duas características básicas: sua ampla distribuição geográfica e, acima de tudo, cronológica. Seria real a permanência de uma mesma "tradição" que se estende do fim do Pleistoceno até as vésperas do período histórico? Tal questionamento tem levado à discussão da validade do conceito de "Tradição Umbu" em termos classificatórios ou mesmo organizacionais, uma vez que tal tradição é geralmente "definida" unicamente pela presença de pontas bifaciais. Portanto, sítios com presença de pontas, existentes em contextos que no Brasil se estendem do Rio Grande

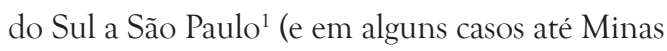
Gerais, conforme Koole, 2007:40), acabam por serem todos associados à Tradição $\mathrm{Umbu}^{2}$. Independentemente da adequação dessa Tradição, é provável que tal associação direta esteja obliterando importantes variações regionais e locais, seja em termos cronológicos, seja espaciais.

\section{Problemas da classificação de vestígios arqueo- lógicos em tradições}

Segundo o PRONAPA (1976:145), tradição é definida como "grupo de elementos ou técnicas que se distribuem com persistência temporal".

A princípio, tais conceitos de tradição eram totalmente desvinculados de qualquer significado "etnográfico" (semelhante ao proposto pelo Midwestern Taxonomic Method, McKern 1939). As pesquisas arqueológicas pioneiras tinham como objetivo a definição de tradições, o que foi feito muitas vezes a partir de um ou dois sítios. Posteriormente, as fases e tradições começaram a ser comparadas a "unidades autônomas e semiautônomas", ou "tribos" ("fases"), "entidades tribais ou lingüísticas” e "nações” "“tradições” - Meggers e Evans 1985; Schmitz 1991).

As críticas à definição e uso do termo "tradição" foram muitas (p.ex.: Dias 2003:51; Dias 2007; Dias e Hoeltz 2010; Hilbert 1994; Milder 1999) e podem ser sintetizadas em alguns aspectos: 1) definição de tradições baseada em um elenco pequeno de critérios tipológicos; 2) o uso de "fósseis-guia" para determinar a associação de determinado sítio a uma ou outra tradição. Em outras palavras, a aplicação do termo "tradição” resultou, por exemplo, no fato corriqueiro de que todos os sítios líticos que apresentassem

(1) Miller Jr. (1972) definiu a Tradição Rio Claro (baseada em sítios da cidade paulista homônima), onde havia a presença de pontas de projétil em algumas das fases. Prous (1991:154) posteriormente relata que essa tradição teria sido englobada dentro da Tradição Umbu. Essa inclusão parece ser bastante controversa, uma vez que nem todas as fases da Tradição Rio Claro apresentam pontas bifaciais e, portanto, não poderiam teoricamente ser incluídas na Tradição Umbu.

(2) Há exceções, veja Chmyz (1981) para uma discussão sobre a Tradição Bituruna. 
pontas bifaciais (o "fóssil-guia") fossem automaticamente classificados como pertencentes à Tradição Umbu.

Passadas mais de três décadas desde as definições iniciais do PRONAPA, tivemos as críticas subsequentes, as remodelações dos conceitos "pronapianos" visando a uma aproximação com a antropologia, uma nova onda de críticas (algumas delas chegando às raias do histrionismo) e, finalmente, chegamos à cristalização do conceito de "tradição", simplesmente porque não há nada para ser colocado no lugar. Após 40 anos de controvérsia, talvez possamos ficar em paz com as tradições, desde que as entendamos como uma ferramenta heurística. Na verdade, talvez a melhor definição de tradição seja a primeva: simplesmente um "grupo de elementos ou técnicas que se distribuem com persistência temporal". Por um feliz acaso, a definição primordial se refere a um grupo, ou seja, um agregado de elementos passíveis de serem listados, e não a uma classe, o que demandaria uma definição (Dunnell 2006). Assim, as tradições jamais serão definidas, apenas descritas. Isso pode ser pouco satisfatório de um ponto de vista formal, mas, novamente, serve perfeitamente a propósitos heurísticos.

Não propomos, portanto, a abolição do uso do termo "Tradição Umbu", porém, ressaltamos a importância de se averiguar quais seriam as fronteiras cronológicas e espaciais dessa tradição, a fim de que não se siga incluindo todos os sítios que apresentem pontas bifaciais nessa categoria. Tradições, ou seja qual for o nome que dermos para esses agregados de fenômenos, podem ser úteis em termos de transmissão de informações entre os profissionais e mesmo para o público leigo. Podem ser deletérias, porém, quando se tornam uma caixa-preta. Nesse contexto, cremos que o (ab)uso do termo "Tradição Umbu" tenha que passar por uma maior reflexão.

Por que estudar pontas? Artefatos líticos formais e suas potenciais abordagens estatísticas

Binford $(1977 ; 1979)$ sugeriu que a organização tecnológica pode ser vista como um contínuo de casos centralizados na produção de ferramentas altamente modificadas e casos de total expediência. No entanto, uma rápida revisão bibliográfica aponta claramente para uma maior ênfase na caracterização e estudo das indústrias líticas formais, seja no Velho Mundo, seja no Novo Mundo. Certamente, há ainda hoje uma fascinação pelas indústrias líticas formais, reminiscente do colecionismo e dos gabinetes de curiosidades, uma vez que os artefatos apresentam maior apelo visual e estético, alem de serem mais facilmente reconhecidos. Outro ponto importante é que, tendo em vista as discussões em torno de estilo e função (Binford 1977; 1979; Dunnel 1978; Sacket 1985), os artefatos formais são mais facilmente "apreendidos" como significantes e significado de processos de transmissão cultural. Isso levou à exploração e desenvolvimento de vários sistemas de classificação, resultando em uma maior disponibilidade de análises estatísticas que podem ser aplicadas de modo a caracterizar tais indústrias. Esse é o caso da Europa e da América do Norte, por exemplo, onde a ênfase ocorre na variação formal entre "tipos" de artefatos, os quais são geralmente considerados independentes da tecnologia. Tal ênfase foi a marca da abordagem da análise lítica desenvolvida por François Bordes (1950), e essa perspectiva tem permeado o pensamento arqueológico muito além da sua aplicação original nas assembleias do Paleolítico Médio. Atualmente, muitos arqueólogos podem não compartilhar a ideia primordial de Bordes, que buscava determinar etnicidade e interação social entre culturas separadas, mas ainda hoje forma e técnica são consideradas como entidades independentes e tecnologia é geralmente considerada como relativamente não informativa (Draper 1985). Dessa forma, não se pode ignorar o potencial explanatório dos artefatos formais, uma vez que essa característica, a normatização de gestos e técnicas visando à produção de artefatos com formas específicas, permite que sejam traçadas relações de interação cultural.

Conforme Dias (2003:225) ressalta, uma indústria lítica só pode ser plenamente entendida com a análise de toda a cadeia operatória, e a variação tipológica observada nos artefatos formais é apenas a ponta do iceberg (Perlés 
1992:223-224). No entanto, o que se propõe no presente artigo não é um aprofundamento do estudo da tecnologia lítica em si, mas sim a apresentação de uma abordagem complementar, envolvendo o uso de ferramentas estatísticas não muito frequentemente utilizadas na Arqueologia brasileira, mas cujo potencial tem sido explorado com sucesso em vários estudos realizados no exterior. $\mathrm{O}$ uso de métodos estatísticos multivariados deverá ajudar a elucidar questões antigas a respeito da caracterização da Tradição Umbu, dos possíveis significados relacionados às mudanças na forma das pontas ao longo do tempo e das relações cronoespaciais entre diferentes conjuntos de pontas, entre outras questões.

\section{Materiais e métodos}

A fim de caracterizar a morfologia das pontas bifaciais, foram realizadas medidas lineares com paquímetro. A Figura 1 mostra os pontos a partir dos quais foram tomadas medidas lineares.
1. Comprimento máximo medial $(\mathrm{AB})$ : projetar até a porção mais distal do pedúnculo, em caso de concavidade

2. Comprimento medial do corpo (AC)

3. Comprimento medial do pedúnculo (CB): projetar até a porção mais distal do pedúnculo, em caso de concavidade

4. Largura aleta-aleta (ED)

5. Largura pescoço (HI)

6. Espessura metade corpo

7. Espessura pescoço (C)

8. Espessura metade pedúnculo

9. Espessura máxima medial (qualquer ponto)

10. Comprimento do ápice $(\mathrm{A})$ até o local de espessura máxima medial

A Tabela 1 apresenta a amostra incluída nas análises de dados métricos e a Figura 2 apresenta as regiões de cada grupo incluídas nessas análises.

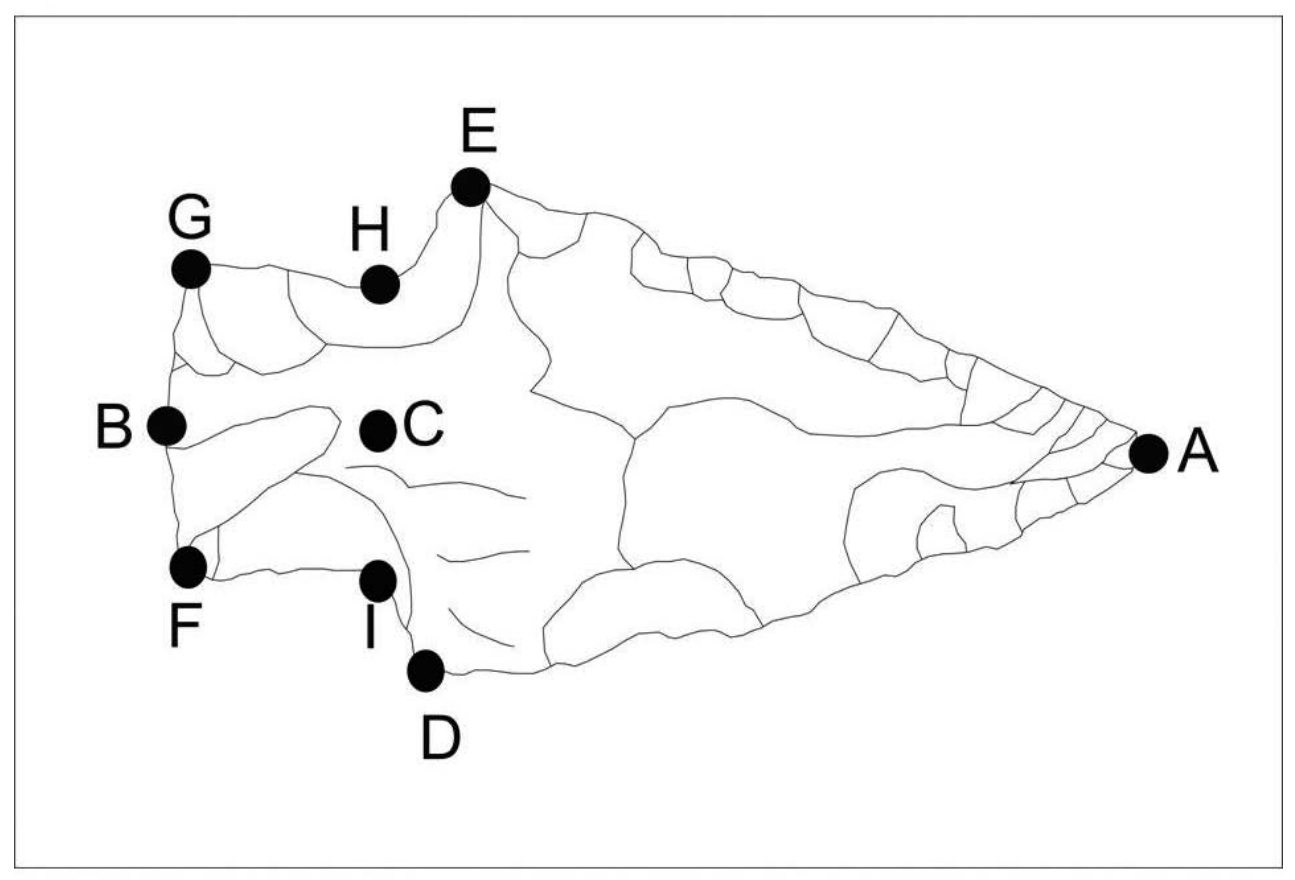

Figura 1: Ponta bifacial exemplificando os pontos a partir dos quais foram tomadas medidas lineares e também os pontos de referência das análises de morfometria geométrica. 


\begin{tabular}{|c|c|c|c|}
\hline Estado & Grupo & Regiões & $\#$ \\
\hline \multirow[t]{3}{*}{$\mathrm{SP}$} & Rio Claro & Rio Claro & 50 \\
\hline & & Ipeúna & 2 \\
\hline & Total & & 58 \\
\hline PR & Reserva & Reserva & 106 \\
\hline \multirow[t]{4}{*}{$\mathrm{SC}$} & Taió & Taió & 42 \\
\hline & & Ibirama & 2 \\
\hline & & Lontras & 2 \\
\hline & Total & & 46 \\
\hline \multirow[t]{6}{*}{ SC } & Urussanga & Urussanga & 28 \\
\hline & & Orleans & 4 \\
\hline & & Criciúma & 1 \\
\hline & & Içara & 2 \\
\hline & & Nova Veneza & 1 \\
\hline & Total & & 36 \\
\hline RS & Dalpiaz & Maquiné & 76 \\
\hline $\mathrm{RS}$ & SAPatrulha & Santo Antônio da Patrulha & 43 \\
\hline \multirow[t]{4}{*}{ RS } & Caí & Caí & 27 \\
\hline & & Brochier & 2 \\
\hline & & Bom Princípio & 1 \\
\hline & Total & & 30 \\
\hline RS & Ivoti & Ivoti & 96 \\
\hline \multirow[t]{2}{*}{$\mathrm{RS}$} & Garivaldino & Taquari & 110 \\
\hline & Total & & 765 \\
\hline
\end{tabular}

Tabela 1: Amostra utilizada para análises com dados métricos. Os nomes de grupos em negrito referem-se a grupos compostos exclusivamente por pontas de um único sítio arqueológico, neste caso, optou-se por nomear o grupo de acordo com o nome do sítio arqueológico.

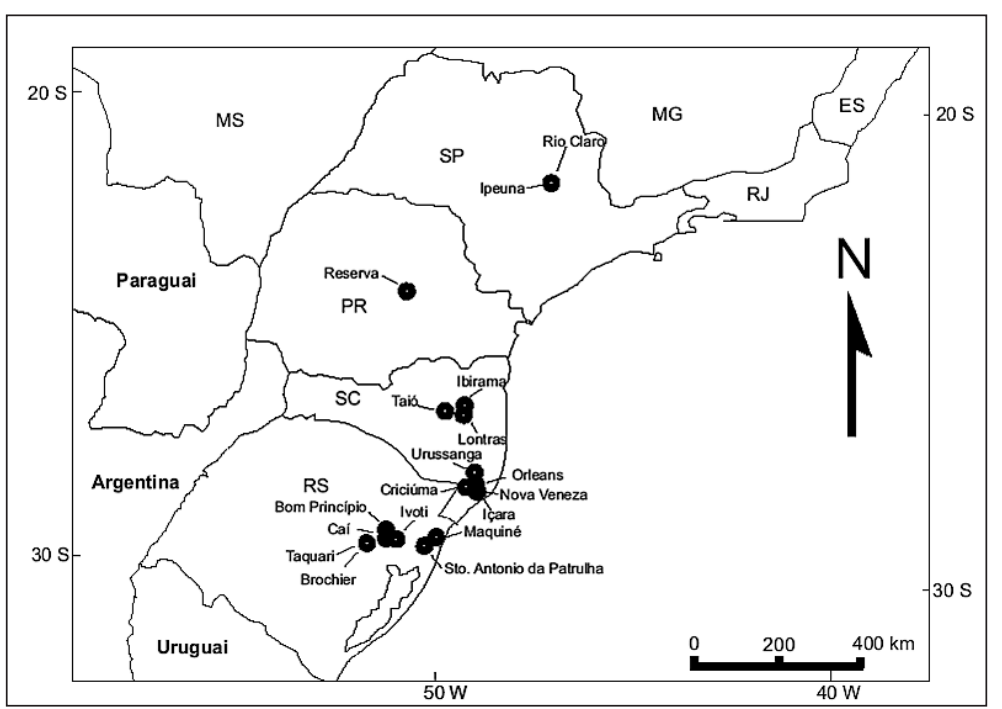

Figura 2: Mapa apresentando as regiões de cada grupo incluído nas análises de dados métricos. 
A análise da forma sempre teve um importante papel em distintas áreas do conhecimento, incluindo biologia, engenharia, entre outras. Diferenças na forma podem ser descritas sumariamente através de objetos familiares, como formas geométricas ou letras do alfabeto. Entretanto, tais descrições são bastante vagas, inacuradas ou até errôneas, especialmente quando as formas são complexas. Embora abordagens utilizando medidas lineares sejam bastante utilizadas para a caracterização da morfologia de artefatos formais, sabe-se que parte importante da informação, especialmente no que diz respeito à forma (em comparação com o tamanho), é perdida. A fim de tentar resgatar a informação sobre a forma, incluiu-se uma análise utilizando a morfometria geométrica. A morfometria geométrica é simplesmente um método quantitativo de se estabelecer comparações de forma (Zelditch et al. 2004). Em outras palavras, a morfometria quantifica a variação e testa as diferenças na forma. A aplicação da morfometria geométrica em dados arqueológicos tem ganhado adeptos em muitos países como os EUA, Inglaterra e Argentina (Buchanan et al. 2007; Buchanan e Collard 2010; Cardillo 2006; 2009; 2010; Castiñera et al. 2009; 2011; Franco et al. 2009; Charlin e González-José 2012), porém, permanece inédita no Brasil. Neste artigo, exploramos algumas das potenciais aplicações deste método na análise da morfologia de pontas bifaciais.

A morfometria quantifica e testa as diferenças na forma através do uso de pontos de referência (Bookstein 1991), também conhecidos na literatura internacional como "landmarks". Neste estudo, os pontos de referência utilizados foram cinco (indicados na Figura 1): o ápice da ponta (A), a ponta da aleta (D), o ponto de encontro entre pescoço e corpo da ponta (I), a lateral do pedúnculo $(\mathrm{F})$ e a extremidade do pedúnculo na linha longitudinal (B). Esses cinco pontos de referência foram digitalizados com o software TPSDig2 em fotografias padronizadas (com a câmera paralela à ponta bifacial e com uma escala em $\mathrm{cm}$ ) em 658 pontas de São Paulo, Paraná, Santa Catarina e Rio Grande do Sul. A descrição de cada grupo analisado encontra-se na Tabela 2 e a Figura 3 apresenta a localização geográfica dos grupos.

\begin{tabular}{|l|l|l|l|c|}
\hline \multicolumn{1}{|c|}{ Estado } & \multicolumn{1}{|c|}{ Grupo } & Sigla & \multicolumn{1}{c|}{ Regiões } & $\#$ \\
\hline São Paulo (SP) & Alice Boer & Ali & Rio Claro & 47 \\
\hline & Ipeúna & Ipê & Ipeúna & 28 \\
\hline Paraná (PR) & Reserva & Res & Reserva & 108 \\
\hline S. Catarina (SC) & Taió & Tai & Taió & 43 \\
\hline & Criciúma & Cri & Criciúma & 35 \\
\hline & Campos Novos & Cpo & Campos Novos & 9 \\
\hline Rio Grande do & Caí & Cai & Caí & 35 \\
\hline Sul (RS) & Capivara & Cap & Ivoti & 99 \\
\hline & Dalpiaz & Dal & Maquine & 75 \\
\hline & Garivaldino & Gar & Taquari & 101 \\
\hline & Ibicuí & Ibi & Ibicuí & 15 \\
\hline & N. Petrópolis & Npe & N. Petrópolis & 9 \\
\hline & Sinos & Sin & Sinos & 17 \\
\hline & Toca Grande & Toc & Sinos & 37 \\
\hline Total & & & & 658 \\
\hline
\end{tabular}

Tabela 2: Origem e número de pontas incluídas na análise de morfometria geométrica. 


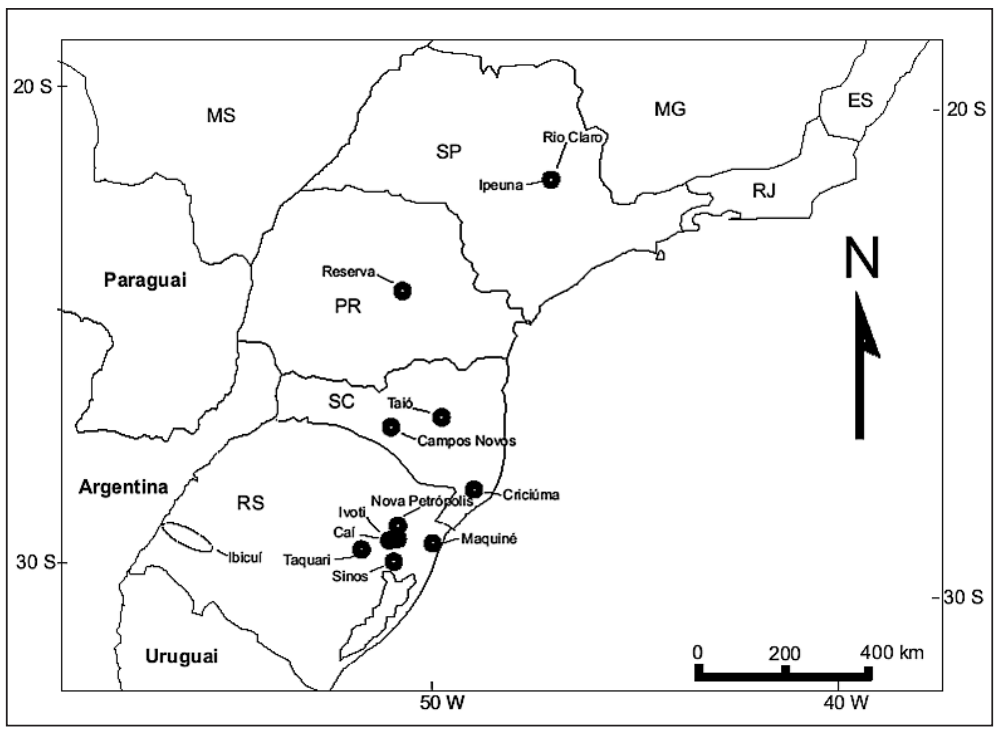

Figura 3: Mapa apresentando as regiões de cada grupo incluído nas análises de dados de morfometria geométrica.

As análises de morfometria geométrica foram feitas com os softwares TPSRegr, TPSSmall, TPSRelw e TPSPLS (Rohlf, s.d.).

\section{Resultados}

A Tabela 3 mostra os resultados da análise de correlação. Os números correspondem àqueles listados na seção de Materiais e Métodos. Todas as variáveis apresentaram-se significativamente correlacionadas $(p<0,05)$. Optou-se por selecionar aquelas variáveis cuja correlação fosse menor que 0,60 (em negrito) e que representassem algumas das dimensões de interesse: comprimento do corpo (2), comprimento do pedúnculo (3), largura do pescoço (5) e espessura do pescoço (7).

Ao fim desta seleção, realizou-se a inspeção da distribuição normal das variáveis em cada amostra, devido à normalidade ser um pré-requisito para a aplicação de diversas análises estatísticas. Utilizou-se para tanto o "Z-score", que consiste no cálculo baseado no valor de cada variável de cada indivíduo subtraído da média (calculada a partir dos valores de todos os indivíduos para essa variável) e dividido por seu desvio padrão. Valores maiores que 4,0 e menores que $-4,0$ foram considerados como "outliers" e excluídos. Foram eliminados 11 indivíduos, sendo seis de Rio Claro e cinco de Reserva. A Tabela 1 (vide Materiais e Métodos) apresenta a amostra utilizada para as análises após a remoção dos indivíduos "outliers".

A seguir, fez-se uma análise de Kruskall-Wallis (versão não paramétrica da ANOVA para amostras independentes, calculada baseando-se na soma dos rankings dos grupos combinados). O objetivo foi verificar se a hipótese de que não há diferenças entre os grupos pode ser rejeitada. Para todas as quatro medidas, foi obtido um valor de $\mathrm{p}<0,000$, o que significa que é possível rejeitar a hipótese nula de que não há diferenças importantes entre as séries.

A Figura 4 mostra os valores de mínimo e de máximo, os limites de $25 \%$ e $75 \%$ da amostra e a mediana, para o comprimento medial do corpo (Figura 4a), o comprimento medial do pedúnculo (Figura 4b) largura do pescoço (Figura 4c) e espessura do pescoço (Figura 4d). Chama a atenção a maior mediana apresentada pelos espécimes de Rio Claro em relação à medida do comprimento medial do pedúnculo. 


\begin{tabular}{|c|c|c|c|c|c|c|c|c|c|}
\hline & 1 & 2 & 3 & 4 & 5 & 6 & 7 & 8 & 9 \\
\hline 2 & 0.97 & & & & & & & & \\
\hline 3 & 0.58 & 0.38 & & & & & & & \\
\hline 4 & 0.65 & 0.60 & 0.48 & & & & & & \\
\hline 5 & 0.68 & 0.60 & 0.62 & 0.70 & & & & & \\
\hline 6 & 0.46 & 0.43 & 0.35 & 0.23 & 0.54 & & & & \\
\hline 7 & 0.47 & 0.38 & 0.5 & 0.29 & 0.59 & 0.76 & & & \\
\hline 8 & 0.42 & 0.32 & 0.55 & 0.29 & 0.59 & 0.61 & 0.84 & & \\
\hline 9 & 0.55 & 0.50 & 0.41 & 0.27 & 0.56 & 0.92 & 0.85 & 0.68 & \\
\hline 10 & 0.85 & 0.84 & 0.45 & 0.55 & 0.50 & 0.21 & 0.40 & 0.37 & 0.36 \\
\hline
\end{tabular}

Tabela 3: Coeficientes de correlação obtidos para as variáveis métricas. Os números referem-se às medidas lineares descritas no texto.
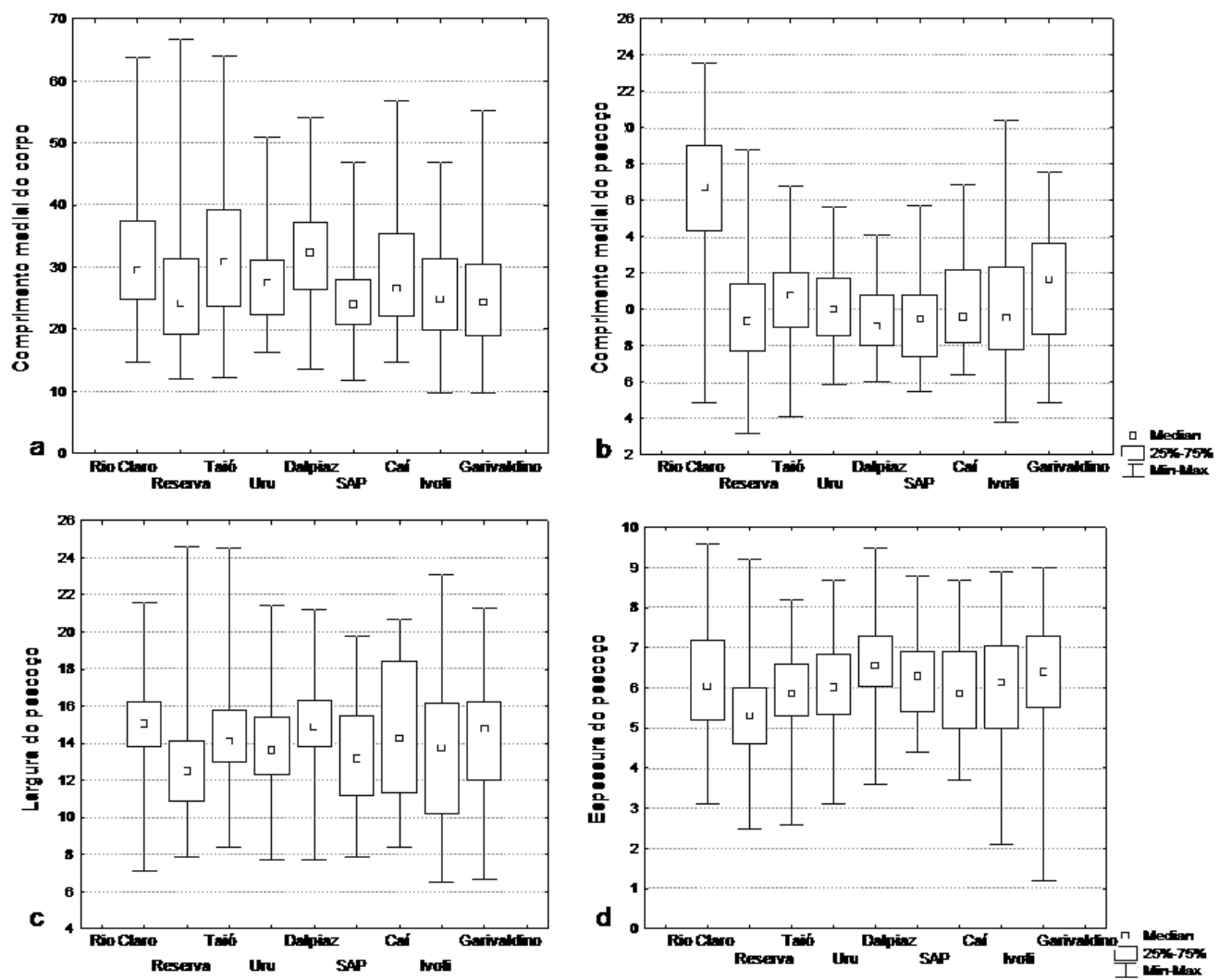

Figura 4: Box-plot apresentando os valores de mínimo e de máximo, os limites de $25 \%$ e $75 \%$ da amostra e a mediana, para o comprimento medial do corpo (a), o comprimento medial do pedúnculo (b), largura do pescoço (c) e espessura do pescoço (d). 
A Análise Discriminante apresentada na Tabela 4 mostra que o grupo com maior porcentagem de classificação correta é o de Rio Claro (SP), seguido de Dalpiaz (RS) e Garivaldino (RS).

A Tabela 5 apresenta os resultados obtidos para os testes de significância entre os grupos. Os valores de $\mathrm{p}<0,05$ (em negrito) mostram que há diferenças significativas entre alguns grupos. Há grupos que são significativamente diferentes de todos os demais, como é o caso de Rio Claro, Dalpiaz e Garivaldino (que apresentaram alta porcentagem de classificação correta na Análise Discriminante, vide Tabela 4).

Reserva também se mostra diferente de todos, exceto Urussanga. Taió é distinto de todos, exceto Urussanga e Caí (o mesmo vale para Caí, que é diferente de todos os grupos, exceto de Urussanga e Taió). Santo Antonio da Patrulha é semelhante a Ivoti e a Urussanga. A Figura 5 apresenta a análise de agrupamento a partir da matriz de distâncias e a Figura 6 apresenta o escalonamento multidimensional a partir da matriz de distâncias. Em ambas as figuras, é possível verificar a grande distância de Rio Claro em relação aos grupos do sul brasileiro.

\begin{tabular}{|l|c|c|c|c|c|c|c|c|c|c|}
\hline & $\%$ & Rio Claro & Reserva & Taió & Urussanga & Dalpiaz & SA Patrulha & Caí & Ivoti & Gariv \\
\hline Rio Claro (SP) & 76.9 & 40 & 5 & 2 & 0 & 0 & 0 & 0 & 0 & 5 \\
\hline Reserva (PR) & 51.9 & 7 & 55 & 4 & 0 & 10 & 0 & 0 & 8 & 22 \\
\hline Taió (SC) & 8.7 & 2 & 21 & 4 & 0 & 5 & 0 & 0 & 3 & 11 \\
\hline $\begin{array}{l}\text { Urussanga } \\
\text { (SC) }\end{array}$ & 0 & 0 & 13 & 1 & 0 & 5 & 0 & 0 & 7 & 10 \\
\hline Dalpiaz (RS) & 63.2 & 0 & 13 & 1 & 0 & 48 & 0 & 0 & 3 & 11 \\
\hline $\begin{array}{l}\text { SAPatrulha } \\
\text { (RS) }\end{array}$ & 0 & 1 & 9 & 0 & 0 & 9 & 0 & 0 & 12 & 12 \\
\hline Caí (RS) & 0 & 1 & 11 & 1 & 0 & 6 & 0 & 0 & 5 & 6 \\
\hline Ivoti (RS) & 10.4 & 2 & 29 & 2 & 0 & 17 & 0 & 2 & 10 & 34 \\
\hline $\begin{array}{l}\text { Garivaldino } \\
\text { (RS) }\end{array}$ & 54.5 & 7 & 21 & 2 & 0 & 9 & 0 & 0 & 11 & 60 \\
\hline Total & 36.5 & 60 & 177 & 17 & 0 & 109 & 0 & 2 & 59 & 171 \\
\hline
\end{tabular}

Tabela 4: Porcentagem de classificação correta dos indivíduos e número de indivíduos classificados em cada grupo.

\begin{tabular}{|l|c|c|c|c|c|c|c|c|}
\hline & $\begin{array}{c}\text { Rio Claro } \\
(\mathrm{SP})\end{array}$ & $\begin{array}{c}\text { Reserva } \\
(\mathrm{PR})\end{array}$ & Taió (SC) & $\begin{array}{c}\text { Urussanga } \\
(\mathrm{SC})\end{array}$ & $\begin{array}{c}\text { Dalpiaz } \\
(\mathrm{RS})\end{array}$ & $\begin{array}{c}\text { SA Patrulha } \\
(\mathrm{RS})\end{array}$ & Caí (RS) & Ivoti (RS) \\
\hline Reserva (PR) & 0.00 & & & & & & & \\
\hline Taió (SC) & 0.00 & 0.00 & & & & & & \\
\hline Urussanga (SC) & 0.00 & 0.12 & 0.08 & & & & & \\
\hline Dalpiaz (RS) & 0.00 & 0.00 & 0.00 & 0.00 & & & & \\
\hline SAPatrulha (RS) & 0.00 & 0.00 & 0.00 & 0.07 & 0.00 & & & \\
\hline Cai (RS) & 0.00 & 0.01 & 0.07 & 0.68 & 0.00 & 0.02 & & \\
\hline Ivoti (RS) & 0.00 & 0.00 & 0.00 & 0.41 & 0.00 & 0.12 & 0.02 & \\
\hline Garivaldino (RS) & 0.00 & 0.00 & 0.00 & 0.01 & 0.00 & 0.00 & 0.00 & 0.02 \\
\hline
\end{tabular}

Tabela 5: Diferenças estatisticamente significativas entre os grupos (em negrito). 


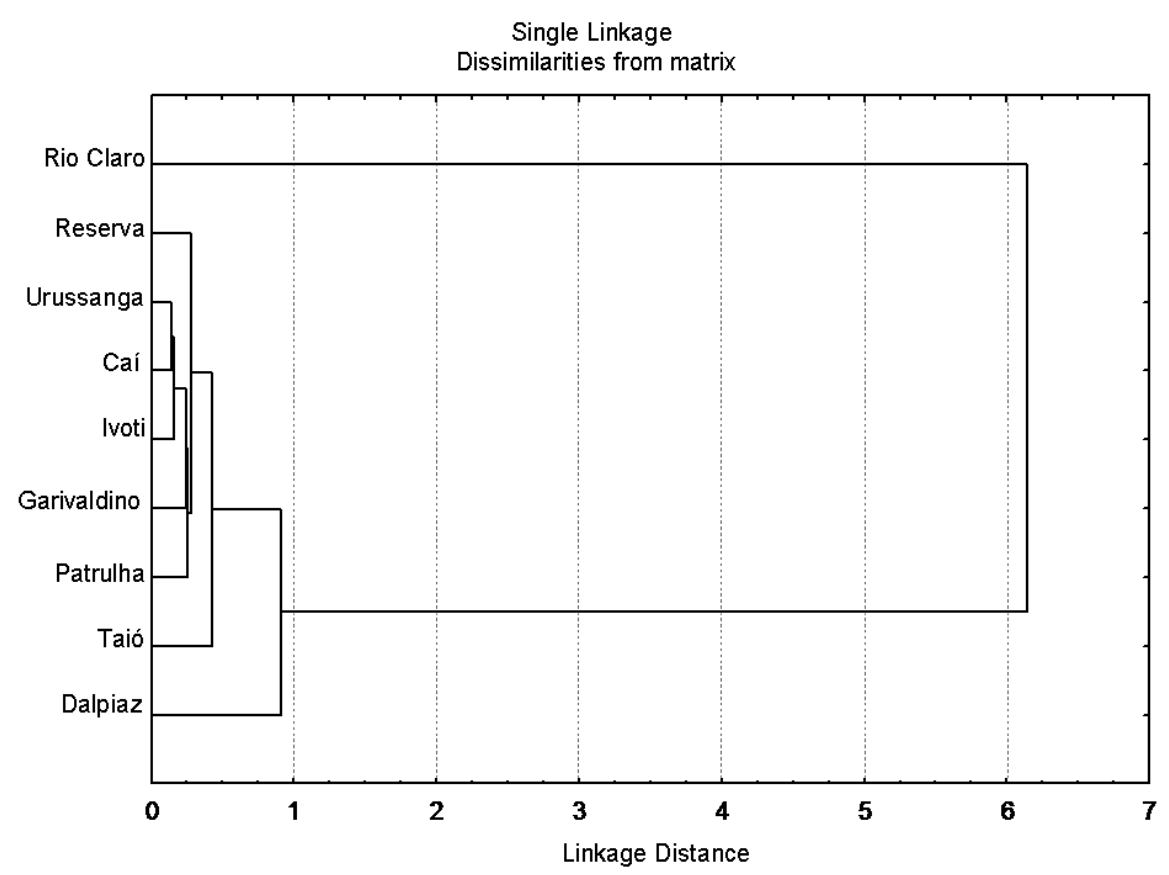

Figura 5: Análise de agrupamento pelo método de ligação simples a partir da matriz de distâncias.

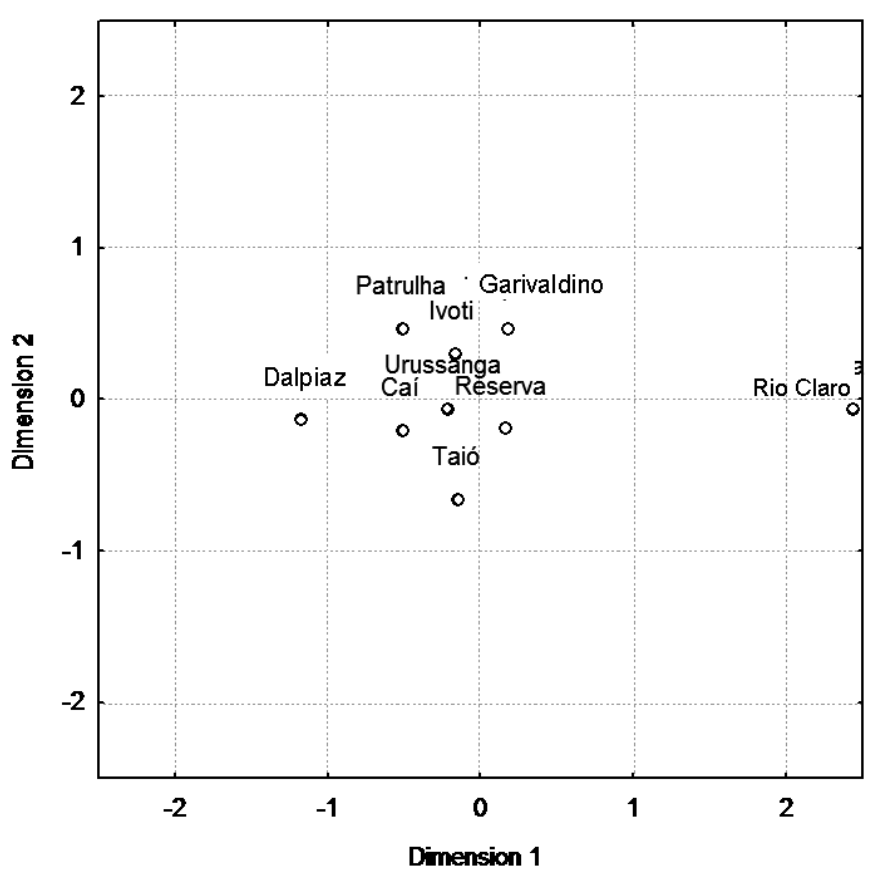

Figura 6: Escalonamento multidimensional a partir da matriz de distâncias. 
Nas análises de morfometria geométrica, o tamanho do centróide mede a dispersão dos pontos de referência em torno do baricentro da configuração (Zelditch et al. 2004). O tamanho do centróide foi computado usando os cinco pontos de referência descritos na seção Materiais e Métodos. Um teste de permutação mostrou que após 1000 "randomizações", havia diferenças significativas entre alguns grupos. A Tabela 6 mostra que os dois grupos de São Paulo (Alice Boer e Ipeúna) são semelhantes entre si e significativamente dife- rentes dos demais $(\mathrm{p}<0.05)$, com exceção de Campos Novos (SC), Ibicuí (RS) e Taió (SC). Observando-se a Figura 7, verifica-se que as pontas de São Paulo são maiores que as do sul, com exceção de dois grupos cuja diferença de tamanho não foi significativa (Campos Novos e Ibucuí).

A correlação entre as distâncias euclidianas e de Procrustes foi de 0.99989, portanto, a aproximação tangencial é boa.

A forma consenso gerada a partir dos dados é apresentada na Figura 8.

\begin{tabular}{|c|c|c|c|c|c|c|c|c|c|c|c|c|c|}
\hline & $\begin{array}{c}\text { Ali } \\
\text { (SP) }\end{array}$ & $\begin{array}{l}\text { Ipe } \\
\text { (SP) }\end{array}$ & $\begin{array}{l}\text { Res } \\
\text { (SP) }\end{array}$ & $\begin{array}{c}\text { Tai } \\
\text { (PR) }\end{array}$ & $\begin{array}{l}\text { Cri } \\
\text { (SC) }\end{array}$ & $\begin{array}{l}\text { Cpo } \\
\text { (SC) }\end{array}$ & $\begin{array}{l}\text { Cai } \\
\text { (SC) }\end{array}$ & $\begin{array}{l}\text { Cap } \\
\text { (RS) }\end{array}$ & $\begin{array}{c}\text { Dal } \\
\text { (RS) }\end{array}$ & $\begin{array}{l}\text { Gar } \\
\text { (RS) }\end{array}$ & $\begin{array}{c}\text { Ibi } \\
\text { (RS) }\end{array}$ & $\begin{array}{l}\text { Npe } \\
\text { (RS) }\end{array}$ & $\begin{array}{c}\text { Sin } \\
\text { (RS) }\end{array}$ \\
\hline Ipe & ns & & & & & & & & & & & & \\
\hline Res & $* * *$ & $* *$ & & & & & & & & & & & \\
\hline Tai & * & ns & ns & & & & & & & & & & \\
\hline Cri & $* * *$ & $* * *$ & ns & * & & & & & & & & & \\
\hline Cpo & ns & ns & * & * & $* * *$ & & & & & & & & \\
\hline $\mathrm{Cai}$ & ** & * & ns & ns & ns & * & & & & & & & \\
\hline Cap & $* * *$ & $* * *$ & ns & $* * *$ & ns & $* * *$ & ** & & & & & & \\
\hline Dal & $* * *$ & $* * *$ & ns & ns & ns & $* * *$ & ns & $* * *$ & & & & & \\
\hline Gar & $* * *$ & $* * *$ & ns & $* * *$ & ns & $* * *$ & $* *$ & $\mathrm{~ns}$ & ** & & & & \\
\hline Ibi & ns & ns & ns & ns & $* *$ & ns & ns & $* * *$ & ** & $* * *$ & & & \\
\hline Npe & $* * *$ & $* * *$ & * & $* * *$ & $* * *$ & $* * *$ & ** & ** & $* * *$ & ** & $* * *$ & & \\
\hline Sin & ** & $* * *$ & ns & * & ns & * & ns & ns & ns & ns & ** & ** & \\
\hline Toc & $* * *$ & $* * *$ & * & $* * *$ & $* * *$ & $* * *$ & $* * *$ & ns & $* * *$ & * & $* * *$ & $* *$ & ns \\
\hline
\end{tabular}

Tabela 6: Valores de $\mathrm{p}$ a partir do teste de Fisher aplicado ao tamanho do centróide. Valores de $\mathrm{p}<0.05={ }^{*}, \mathrm{p}<0.01$ $={ }^{* *}, \mathrm{p}<0.001={ }^{* * *}$, valor não significativo $=$ ns. 
A Figura 9 mostra a Análise de Componentes Principais aplicada às coordenadas de forma. Verifica-se uma considerável sobreposição dos grupos. Também é possível verificar os extremos de forma dessa amostra. Nesse caso, a forma consenso observada na Figura 6 estaria localizada no centro do gráfico (coordenadas 0,0 ). É possível verificar que há pontas cujo corpo é longo e o pedúnculo afilado (formas da porção superior do gráfico), pontas cujo corpo é curto e o pedúnculo afilado (porção direita), pontas cujo corpo é curto e o pedúnculo bifurcado (porção inferior) e ponta cujo corpo é comprido e o pedúnculo bifurcado (porção esquerda).

A partir da matriz dos escores das deformações relativas ("Relative Warps Score Matrix") foi realizada uma análise discriminante. $\mathrm{O}$ gráfico representando as duas funções canônicas dessa análise discriminante (Figura 10) mostra que os dois grupos de São Paulo possuem forma distinta dos grupos do sul. Também é possível verificar uma associação entre Paraná e Santa Catarina, especialmente entre Reserva (PR) e Taió (SC).

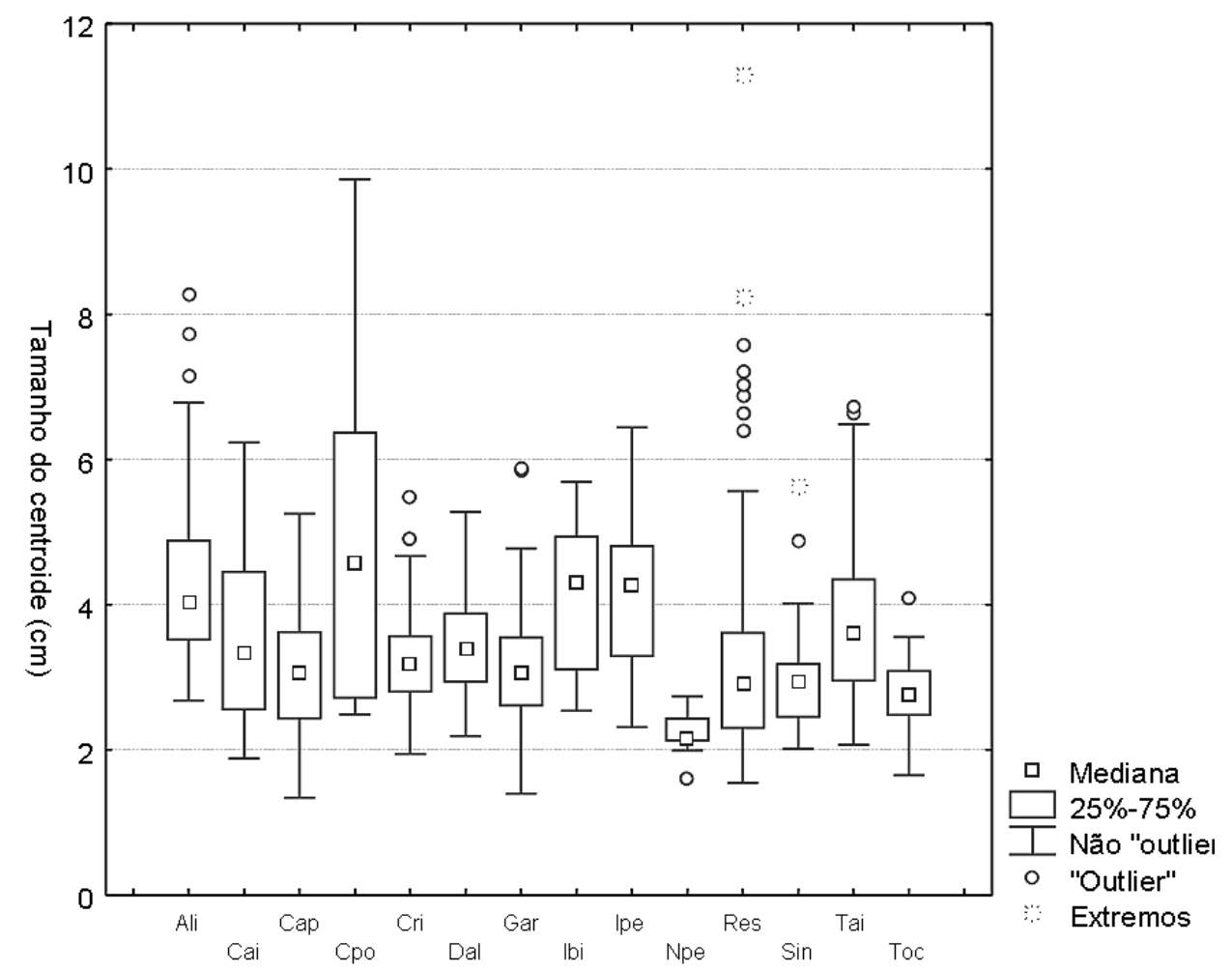

Figura 7: Box-plot apresentando os valores de mínimo e de máximo, os limites de $25 \%$ e $75 \%$ da amostra e a mediana, para o tamanho do centroide em centímetros. 


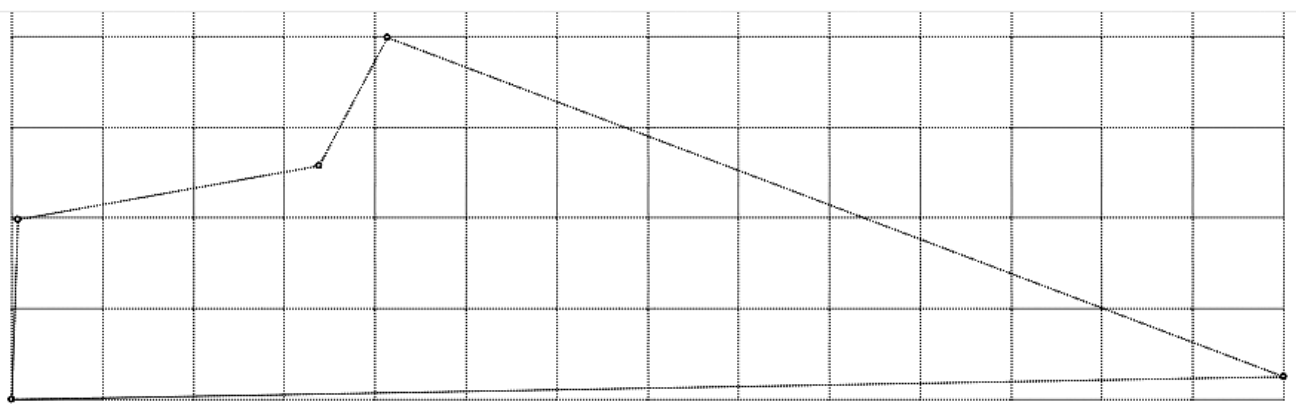

Figura 8: A forma consenso da amostra analisada. Atentar para o fato de apenas metade da ponta estar representada.

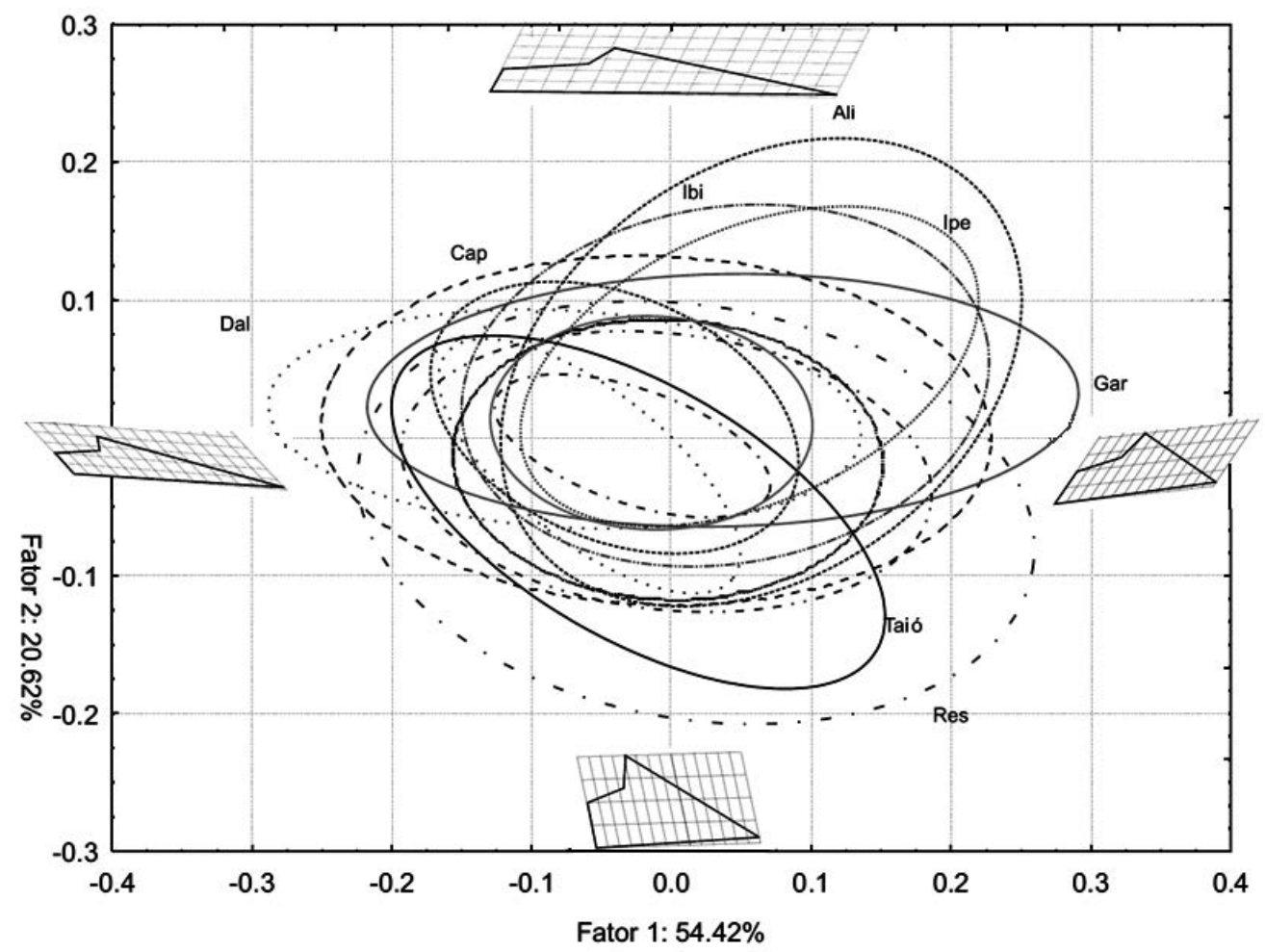

Figura 9: Gráfico dos fatores obtidos a partir da Análise de Componentes Principais e os extremos de variação de forma. 


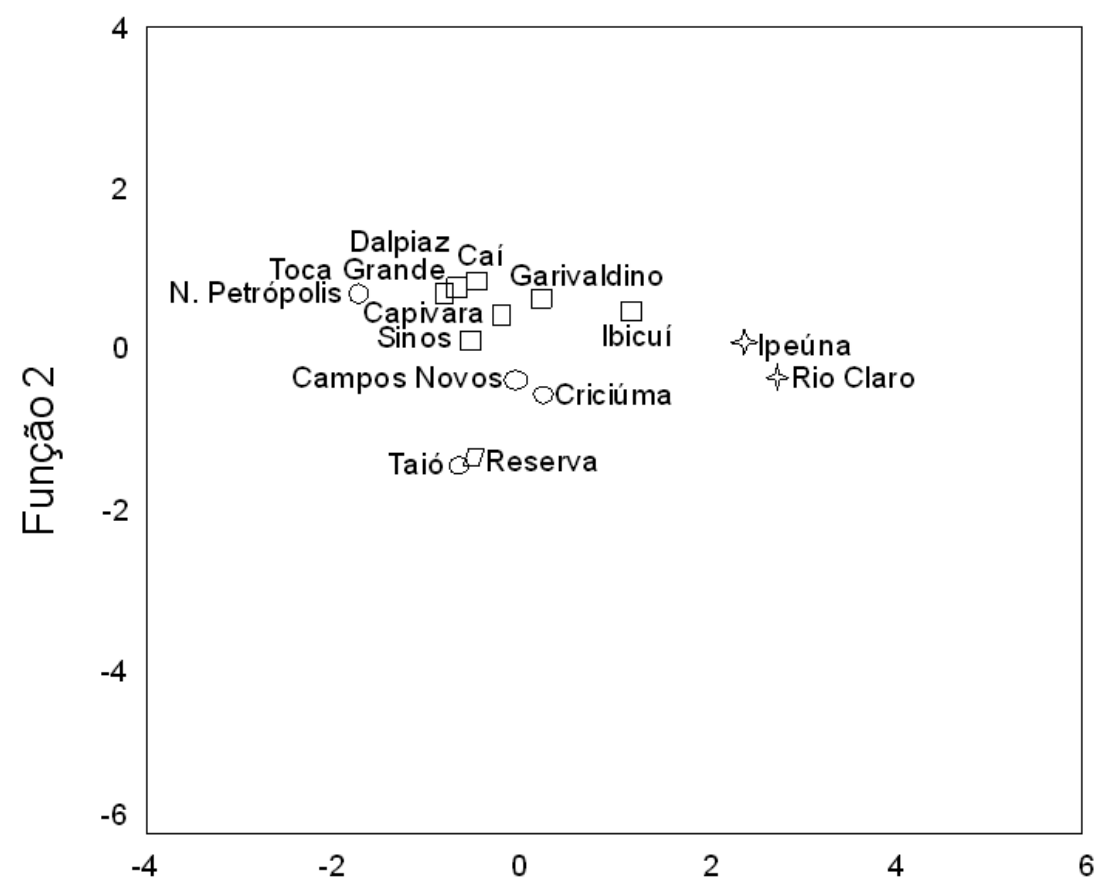

\section{Função 1}

Figura 10: Gráfico mostrando as duas funções resultantes da análise discriminante. Legenda: regiões de São Paulo (estrelas), Paraná (paralelogramo), Santa Catarina (círculos) e Rio Grande do Sul (quadrados).

\section{Conclusões}

Os dados métricos e as análises de morfometria geométrica apontam para diferenças relevantes no tamanho e na forma das pontas de São Paulo em relação às demais. No caso da análise de morfometria, onde foi possível incluir dois grupos paulistas de regiões bastante próximas (Rio Claro e Ipeúna), foi verificada uma grande semelhança entre as pontas desses dois grupos. De modo geral, as pontas de São Paulo parecem ser maiores que as demais pontas estudadas (com exceção de dois grupos sulinos que não apresentaram diferenças significativas em relação a São Paulo). Embora não acreditemos ser necessário ressuscitar a categoria "Tradição Rio Claro”, nossos resultados preliminares apontam para uma diferença importante na morfologia das pontas de São Paulo em relação às pontas do sul do país. Uma vez que a Tradição Umbu foi definida a partir de pontas do sul, as pontas de São Paulo não poderiam ser consideradas parte desse grupo. É possivel que as pontas oriundas de Minas Gerais e Mato Grosso também sejam distintas das pontas da região sul.

Há indícios de similaridade entre as pontas do Paraná e de Santa Catarina, assim como entre alguns grupos do Rio Grande do Sul. Tais similaridades poderiam estar sendo exacerbadas 
devido à enorme diferença que existe entre as pontas de São Paulo e as demais, o que faz com que o sul do Brasil pareça muito homogêneo. Futuras análises, considerando-se apenas o sul do Brasil, intentarão explorar a diversidade dentro dessa região.

\section{Agradecimentos}

Agradecemos a todas as pessoas que contribuíram para o desenvolvimento desta pesquisa, em especial: Adriana Schmidt Dias, Adriana Pereira dos Santos, Afonso Paseto, Cinira Mülk, Daiza Lacerda, Dária Barreto, Dinan Rogério, Dione Bandeira, Jefferson Dias, João Böer, João Messeti, José Donizeti de Souza, Juliano Bitencourt Campos, Maria Antonieta Cassab, Marisa Afonso, Maryzilda Campos, Natália Zanella, Paulo Jacob, Pedro Ignácio Schmitz, Ricardo Coelho, Sérgio Klamt, Silézia Pinto, Teresa Fossari, Waldomiro Malaguti. Agradecemos também o apoio financeiro do CNPq (159776/2010-4) e FAPESP (09/54720-9; 2010/06453-9).

OKUMURA, M.; ARAUJO, A. Bifacial points from Southern Brazil: statistical analysis and cultural implications. R. Museu Arq. Etn., São Paulo, n. 23, p. 111-127, 2013.

Umbu Tradition, distributed in southern and southeastern Brazil, is characterized by the presence of bifacial points and presents dates ranging from 12,700 years BP to the historical period. This tradition is problematic because of its wide geographic and chronological range. This work aims to contribute to the characterization of the morphological variability of points through quantitative analysis. The results show that points made by groups from south-eastern Brazil (São Paulo state) are distinct from those made by the southern groups (Paraná, Santa Catarina and Rio Grande do Sul). This would indicate a regional identity shared only by some groups from São Paulo (at least regarding the projectile points). It is possible that Tradition Umbu presents a more restricted range, both in chronological and spatial terms, than the one proposed today.

Keywords: Lithics, Geometric morphometrics, Umbu Tradition

\section{Referências Bibliográficas}

BINFORD, L. R.

1977 Forty-seven trips: a case study in the character of archaeological formation process. In: Stone tools as cultural markers: change, evolution, and complexity, edited by R. V. S. Wright, pp. 24-36. Australian Institute of Aboriginal Studies, Canberra.
BINFORD, L. R.

1979 Organizational and formation processes: looking at curated technologies. Journal of Anthropological Research 35:255-273.

Bookstein, F. L.

1991 Morphometric tools for landmark data. Cambridge University Press, New York. 
BORDES, F.

1950 Principes d'une méthode d'étude des techniques de débitage et de la typologie du Paléolithique ancien et moyen. L'Anthropologie 54.

BUCHANAN, B.; JOHNSON, E.; STRAUSS, R. E.; LEWIS, P. J.

2007 A morphometric approach to assessing Late Paleoindian projectile point variability on the southern High Plains. Plains Anthropologist 52:279-299.

BUCHANAN, B.; COLLARD, M.

2010 A geometric morphometrics-based assessment of blade shape differences among Paleoindian projectile point types from western North America. Journal of Archaeological Science 37:350-359.

CARDILLO, $\mathrm{M}$.

2006 Explorando la variación en las morfologías líticas a partir de las técnicas de análisis de contornos. El caso de las puntas de proyectil del holoceno medio-tardío de la Puna de Salta (San Antonio de los

CARDILLO, M. Cobres, Argentina). Werken 7: 77:88.

2009 Temporal trends in the morphometric variation of the lithic projectile points during the middle Holocene of Southern Andes (Puna region) - a coevolutionary approach. In Theoretical and Methodological Issues in Evolutionary Archaeology: toward an unified Darwinian paradigm, edited by Hernán J. Muscio, Gabriel E. J. López, pp. 13-20. BAR International Series 1915

CARDILLO, M.

2010 Some applications of Geometric Morphometrics to archaeology. In Morphometrics to Nonmorphometricians (Lecture Notes in Earth Sciences), edited by Ashraf M. T. Elewa, pp. 325-341. Springer, New York.

CASTIÑEIRA, C.; CARDILLO, M.; CHARLIN, J.; FERNICOLA, J. C.; BAEZA, J.

2009 Analisis morfométrico de cabezales líticos "cola de pescado" de la Rep. Oriental del Uruguay. Arqueometría Latinoamericana (2do. Congreso Argentino y 1ro Latinoamericano, Buenos Aires, Argentina) 1:360-366.

CASTIÑEIRA, C.; CARDILLO, M.; CHARLIN, J.; BAEZA, J.

2011 Analisis de Morfometría Geométrica en Puntas Cola de Pescado del Uruguay. Latin American Antiquity 22(3):335-358.
CHARLIN, J.; GONZÁLEZ-JOSÉ, R.

2012 Size and Shape Variation in Late Holocene Projectile Points of Southern Patagonia: A Geometric Morphometric Study. American Antiquity, 77(2):221-242.

CHMYZ, I.

1981 Relatório das pesquisas arqueológicas realizadas na área da Usina Hidrelétrica de Salto Santiago (1979-80). ELETROSUL/ IPHAN, Florianópolis/Curitiba.

DIAS, A. S,

2003 Sistemas de assentamento e estilo tecnológico: uma proposta interpretativa para a ocupação pré-colonial do alto Vale do Rio dos Sinos, Rio Grande do Sul. Tese de doutorado, FFLCH-USP.

DIAS, A. S.

2007 Novas perguntas para um velho problema: escolhas tecnológicas como índices para o estudo de fronteiras e identidades sociais no registro arqueológico. Boletim do Museu Paraense Emilio Goeldi (Ciências Humanas) 2(1):59-76.

DIAS, A. S.; HOELTZ, S.

2010 Indústrias líticas em contexto: o problema Humaitá na arqueologia sul-brasileira. Revista de Arqueologia 23(2):44-72.

DRAPER, N.

1985 Back to the drawing board: a simplified approach to assemblage variability in the Early Palaeolithic. World Archaeology 17(1):3-19.

DUNNELL, R. C.

1978 Style and function: a fundamental dichotomy. American Antiquity 43:192-202.

DUNNEL, R. C.

2006 Classificação em arqueologia. Editora da Universidade de São Paulo, São Paulo. $259 \mathrm{pp}$.

FRANCO, N. V.; CASTRO, A.; CARDILlO, M.; CHARLIN, J.

2009 La importancia de las variables morfológicas, métricas y de microdesgaste para evaluar las diferencias en diseños de puntas de proyectil bifaciales pedunculadas: un ejemplo del sur de Patagonia continental. Magallania (Chile) 37(1):99-112.

HILBERT, K.

1994 Caçadores coletores pré-históricos no sul do Brasil: um projeto para a redefinição das tradições líticas Umbu e Humaitá. In: Negros e indios: literatura e história, edited by Moacyr Flores. EDIPUCRS, Porto Alegre. 
KERN, A. A.

1981 Le précéramique du plateau sud-brésilien. Tese de doutorado, Ecole des Hautes Études en Sciences Sociales (EHESS), Paris.

KOOLE, E. K. M.

2007 Pré-história da província cárstica do Alto São Francisco, Minas Gerais: a indústria lítica dos caçadores-coletores arcaicos. Dissertação de mestrado, Museu de Arqueologia e Etnologia (USP), São Paulo.

MCKERN, W. C.

1939 The Midwestern Taxonomic Method as an aid to archaeological culture study. American Antiquity 4:301-313.

MEGGERS, B. J.; EVANS, C.

1985 A utilização de sequências cerâmicas seriadas para inferir comportamento social. Instituto de Arqueologia Brasileira (Boletim Série Ensaios) 3:1-48.

MILDER, S. E. S.

1999 Caçadores coletores: a problemática arqueológica e ambiental sobre os primeiros povoadores do Rio Grande do Sul. Revista do CEPA (Santa Cruz do Sul) 23:7-56.

MILLER JR., T. O.

1972 Arqueologia da região central do Estado de São Paulo. Dédalo 16:13-118.

NOELLI, F. S.

1999. A ocupação humana na região sul do

2000 Brasil: arqueologia, debates, perspectivas 1972-2000. Revista USP 44:218-269.

PERLÉS, C.

1992 In search of lithic strategies: a cognitive approach to prehistoric chipped stone assemblages. In Representations in Archaeology, edited by Christopher Peebles, Jean-Claude Gardin, pp. 223-247. Indiana

\section{PRONAPA} University Press, Bloomington.

1976 Terminologia arqueológica brasileira para a cerâmica - segunda edição revista e ampliada. Cadernos de Arqueologia (UFPR) 119-148.

PROUS, A.

1991 Arqueologia brasileira. Universidade de Brasília, Brasília.

ROHLF, F. J.

s.d. Tps Series. Department of Ecology and Evolution, State University of New York, Stony Brook, New York. http://life.bio. sunysb.edu/morph/

SACKETT, J. R.

1985 Style and ethnicity in the Kalahari: a reply to Wiessner. American Antiquity 50:154159.

SCHMITZ, P. I.

1984 Caçadores e coletores da Pré-historia do Brasil. Instituto Anchietano de Pesquisas, Unisinos, São Leopoldo.

SCHMITZ, P. I.

1987 Prehistoric hunters and gatherers of Brazil. Journal of World Prehistory 1(1):53-126.

SCHMITZ, P. I.

1991 Migrantes da Amazônia: a Tradição Tupi-guarani. Pré-História do Rio Grande do Sul - Documentos 5:31-66.

SCHMITZ, P. I.

1999 A questão do Paleoíndio. In: Pré-História da Terra Brasilis, edited by Maria Cristina Tenório, p. 55-59. Editora UFRJ, Rio de Janeiro.

SCHMITZ, P. I.; BARBOSA, A. S.; RIBEIRO, M. B.

1980 Temas de Arqueologia Brasileira 1 - Paleoíndio. Anuário de Divulgação Científica, Goiânia, Universidade Católica de Goiás 5, $99 \mathrm{pp}$.

ZELDITCH, M. L.; SWIDERSKI, D. L.; SHEETS, H.

D.; FINK, W. L.

2004 Geometric morphometrics for biologists: a primer. Elsevier Academic Press, New York. 